Research Article

\title{
The Design and Implementation of Test System Based on Programmable Excitation Power Supply for Mining Comprehensive Protector
}

\author{
Tian-ye Liu ${ }^{1,2^{*}}$ and Zhi-jie Zhang ${ }^{1,3}$ \\ ${ }^{1}$ Instrumentation Science \& Dynamic Measurement Key Laboratory of Ministry of Education (North University of China), Taiyuan, \\ 030051, China \\ ${ }^{2}$ School of Computer and Control Engineering, North University of China, Taiyuan, 030051, China \\ ${ }^{2}$ School of Instrument and Electronics, North University of China, Taiyuan, 030051, China
}

Received 15 September 2013; Accepted 23 November 2013

\begin{abstract}
As comprehensive protectors for coal mining (referred to comprehensive protectors) in use are prone to fail, it can timely screen out the invalid comprehensive protector by periodic functional test when it is used (it is called test in use) to ensure the production safety. The test in use needs the specialized test equipment, which is not used in delivery inspection by the manufacturers of comprehensive protectors. Thus, testing excitation power becomes a constraint for the improvement of the accuracy of test in use and the degree of automation. To solve the problem, this paper developed a power frequency programmed input-output testing excitation power supply, and on that basis it also realized the mining comprehensive protector test system in use with the excitation circuit and voltage program-controlled output.
\end{abstract}

Keywords: Mining Comprehensive Protector, Test In Use, Programmed Input-output Excitation Power Supply

\section{Introduction}

It can timely screen out the invalid comprehensive protector by the periodic functional test for mining comprehensive protector in use. It can ensure comprehensive protector be in a valid and accurate state of the protection functions for mining electromechanical device electric faults by the test and so it plays an important role in ensuring the safety of coal production. Now the periodic test adopts off-line mode rather than on-line mode. Moreover, it is different from delivery inspection by manufacturers of the comprehensive protector, which is called comprehensive protection test in use. Comprehensive protector for coal mining is the relay protection device equipped by all kinds of electrical equipments in underground coal mining district. It is used for supervision and protection of the underground electrical equipment short-circuit, overload, over voltage, low voltage, leakage, loss of phase and other electrical faults, which is the last technical safety measure in the operation of electrical equipment in coal mines[1],[2],[3],[4]. For the reasons in literature [5],[6], the reliability of comprehensive protector in use is not high. Therefore, it is necessary to do periodic testing and inspection for comprehensive protector in use [7]. Carrying on testing and inspection of comprehensive protector in use needs to rely on a certain type of test equipment.

The economy, operator requirements, testing requirements and other aspects of the test equipment are different from the test equipments used by researchers, manufacturers and social professional testing organizations of the comprehensive protector. The advance of computer technology enables the development of a test device of the comprehensive protector in use [8]. Some test equipments of comprehensive protectors in use have been developed. For example, Huainan Institute adopted computer technologyusing mono-plate professor as a testing device controllerimplementing the quantitative test of comprehensive protectors [9]. North University of China developed a PCbased test equipment which had solved the problems of incomplete test projects and test versatility [10],[11],[12]. The literature review [13] did the modeling research of a comprehensive protector test system. These studies and the practical use of those test systems which they developed in mines play a significant role in the advance of the comprehensive protector test in use.

The above studies solve the problem of the test versatility of comprehensive protectors in use. What's more, the accuracy of the test results and the degree of automation are also greatly improved. But as far as we know, all power frequency test excitation signal sources of the test equipments coming into service adopt the contact voltage regulator, in which there are the following deficiencies: long response lag time of excitation signal, low signal accuracy, poor output stability influenced by the variation of the power supply side and load side (test object); the weakness of excitation signal accuracy reduces the accuracy of test results; excitation signal needs to be manually adjusted[14]; the high artificial participation during the test process hinders 
the improvement of the degree of test automation. To cope with these shortcomings, this paper developed a power frequency programmed input-output testing excitation power supply based on direct digital synthesis technology. It was able to shorten the signal response time, to improve stability of the output by the feedback control and to realize automatically adjust through the programmable controlling output. Based on the programmable power supply, this paper implemented a full automatic comprehensive protector test system in use for coal mining, which ensures the test accuracy and improves the degree of automation of the testing process.

\section{Test Principle}

The test requirements for mining comprehensive protectors in use are: economic test device, low test cost; high efficiency, less time for test, test qualified comprehensive protectors timely returning to work underground; achieving the purposes of initially screening out the invalid comprehensive protectors, not having to test all items as the comprehensive protector manufacturers do and test accuracy can also be appropriately reduced. The very small part of the comprehensive protectors with uncertain test results can be sent to the manufacturers or social professional testing organizations for final test.

For the test requirements that comprehensive protectors in use are a kind of functionality confirmatory test, test methods adopt simulation test. The test principles are as follows: test system simulates the work signal that the electrical equipment equipped to comprehensive protectors works in the condition of normality and various fault as the excitation signal, thus simulating the actual electrical environment that comprehensive protector runs, and then measuring the corresponding response signal and investigating the relationship between stimulus and response to determine whether the protection performance of the comprehensive protector is qualified.

\section{Signal Analyses for Test System}

The test system needs to provide three-phase AC voltage and three-phase alternating current that electrical equipments equipped to comprehensive protectors works in the normal and various fault conditions. As comprehensive protector is connected with the protected electrical equipment by voltage and current transformers, the test device does not have to provide a corresponding primary high voltage (up to thousands of volts) or large current (to several thousand amperes). It just needs to provide secondary low voltage and current of the voltage and current transformer. But for the amplitude, phase, frequency of every phase need to be adjusted independently. According to reference [11], the required voltage and current parameters are shown in the following Table1.

There are also switching value output signal and resistance signal used to test leakage and insulation in addition to voltage and current signals that can be used as test excitation signal. Switching value output signals are used to simulate block and control signal that transmitted to comprehensive protectors, while parts of these are used for test process control. Both of them require that the logic relationship of switching value output signal is programmable. Resistance signal is used to simulate insulation of electrical equipments equipped to comprehensive protectors. It requires continuous adjustment and the adjustment range is between 0 and $50 \mathrm{~K} \Omega$.
In addition, in order to achieve the test function, some signals need to be measured. They are mainly the response signal of the comprehensive protector, including whether the relay status is changed (switching value input signals), the delay time of response and the voltage, current or resistance values when the relay status is changed.

Table 1 Power frequency excitation signal parameters

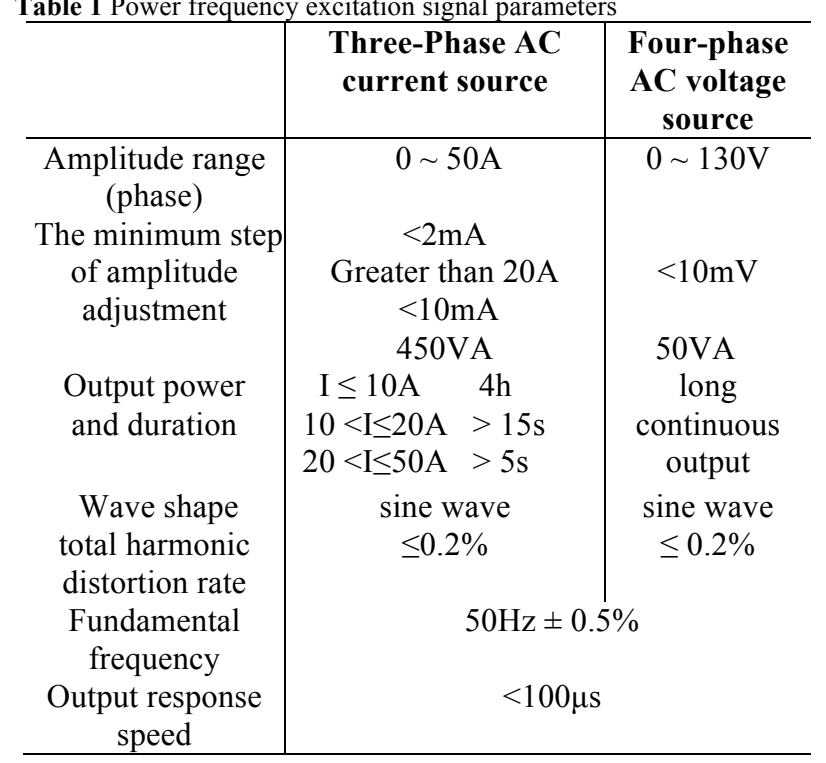

The requirement of switching value input signal interface is able to withstand of inputting maximum voltage no less than $250 \mathrm{~V}$ (DC), and the signal interface can also adapt to the intake of charged or no charged pots with different amplitude and polarity. The switching value input contact circuit used to measure the action value and the return value requires fast action, and relay operating time should not exceed $10 \mathrm{~ms}$ [15].

Measurement time is ranged from a few milliseconds to 10 minutes. When the measurement time is greater than $1 \mathrm{~s}$, the relative error is less than 5/1000. And when the measurement time is less than $1 \mathrm{~s}$, the resolution of the instrument measuring time should be $0.1 \mathrm{~ms}$ [15].

\section{Programmed Input-output Excitation Power Supply Design}

\subsection{Power Supply Scheme design}

The programmed input-output power supply is comprised of programmed input-output signal source and power amplifier. Programmed input-output signal source is realized by adopting sine wave digital direct synthesis (referred to DDS) technology with digital frequency modulation, phase modulation, amplitude modulation, which are keys to achieve programmed output.

DDS is a kind of frequency synthesis technology, which starts from the phase and directly uses digital technology to generate waveform. The fully digital architecture of DDS device is easy for integration, which has the ability to tightly integrate computer technology. Moreover, the programmable regulation of frequency, phase, and amplitude can be achieved.

The principle structure of programmable sine wave power is shown in Fig.1.

In Fig. $1, \mathrm{~K}$ is the frequency control word, $\mathrm{P}$ the phase control word, $\mathrm{M}$ the amplitude control word, fc the reference clock frequency, $\mathrm{N}$ the word length of the phase accumulator 
and also the word length of phase control word P, D the word length of waveform RAM data and D / A converter.

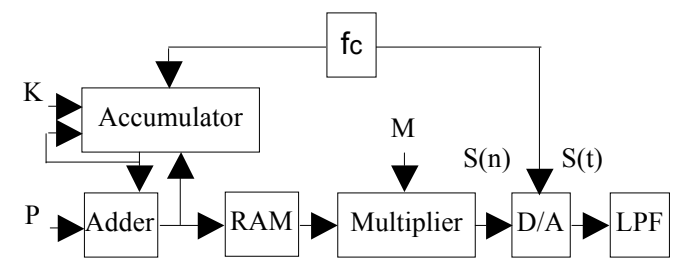

Fig.1 Schematic diagram of programmable sine wave power supply

According to the requirements, after the digital discrete of sinusoidal base wave and superimposed harmonic wave, the CPU stores the values in waveform RAM. When outputting the signal, under the control of clock fc, the phase accumulator does accumulate in $\mathrm{K}$ steps. The N-bit binary output added to phase control word $\mathrm{P}$, making this value as the address of waveform RAM. After addressing the waveform RAM, multiplying the output of waveform RAM with amplitude control word $\mathrm{M}$ and obtaining the amplitude code $\mathrm{S}(\mathrm{n})$ of $\mathrm{D}$ bit. By D/A converter, $\mathrm{S}$ (n) turns into a stepped wave $S(t)$, and through the low-pass filter $S(t)$ is smoothed to obtain a continuous synthesis sine wave signal. As voltage and current signal, it is inputted to the power amplifier for power amplification, thus acquiring output signals that the waveform, phase, amplitude and power meet the requirements.

\subsection{Main parameters selection}

(1)Referencing clock frequency $f_{c}$ determines the maximum frequency of output signal.

According to the formula $f_{\text {o.max }} \leq 0.4 f_{c}, f_{\text {o.max }}$ in this application is 20 harmonics with frequency of $50 \mathrm{~Hz}$, which is $1000 \mathrm{~Hz}$, so

$$
f_{c} \geq 2.5 k H_{Z} \times 1000 H_{Z}=2.5 k H_{Z}
$$

If selecting $f_{c}$ as above, there are only two synthesis points per period when outputting the maximum output signal which cannot guarantee the quality of the signal waveform. According to this, to improve the waveform accuracy of the output signal and make the minimum number of synthesis points per period (i.e. highest frequency output) 2000, selecting the reference clock frequency is:

$$
2.5 k H_{Z} \times 2000=5 M H_{Z}
$$

At this moment, outputting $1000 \mathrm{~Hz}$ sine wave signal is analyzed by the signal analyzer to meet the total harmonic distortion requirements.

(2) The word length $N_{k}$ of frequency control word K determines the frequency of the output signal to adjust the minimum step size.

$$
\begin{aligned}
& \because \Delta f \geq f_{c} / 2^{N_{K}} \\
& \therefore 2^{N_{K}} \geq f_{c} / \Delta f=5 \times 10^{6} / 0.001=5 \times 10^{9} \\
& \therefore N_{K}=64
\end{aligned}
$$

(3) The word length $N_{P}$ of phase control word $\mathrm{P}$ determines the phase adjustment fineness of the output signal.

$$
\begin{aligned}
& \because \Delta P \geq 360^{\circ} / 2^{N_{P}} \\
& \therefore 2^{N_{P}} \geq 360^{\circ} / \Delta P=360^{\circ} / 0.1^{\circ}=3600 \\
& \therefore N_{P}=12
\end{aligned}
$$

(4) The adjustment of the signal source amplitude adopts 16-bit D/A converter, which makes the adjustment fineness less than $0.01 \%$ of full scale.

(5) Power amplifiers adopt linear modulation amplifier, and designed with short-circuit protection in voltage circuit, broken-circuit protection in circuit and fast current-limiting protection, in order to ensure long-term stable and reliable power amplifier work.

\subsection{Output Performance Parameters}

The main performance parameters of the programmable power supply output are shown in Table 2 .

Table 2 Main performance parameters of programmable power supply output

\begin{tabular}{c|c|c}
\hline & Voltage output & Current output \\
\hline $\begin{array}{c}\text { Output range } \\
\text { Adjustment } \\
\text { fineness }\end{array}$ & $3 \times 0 \mathrm{~V} \sim 380 \mathrm{~V}$ & $3 \times 0.1 \sim 100 \mathrm{~A}$ \\
$\begin{array}{c}\text { Maximum output } \\
\text { capacity per } \\
\text { phase }\end{array}$ & $0.01 \%$ full scale & $0.001 \mathrm{~A}($ minimum) \\
$\begin{array}{c}\text { Adjustment } \\
\text { range }\end{array}$ & $0-120 \%$ & $500 \mathrm{VA}(100 \mathrm{~A})$ \\
$\begin{array}{c}\text { Output power stability } \\
\text { Coefficient of output waveform } \\
\text { distortion }\end{array}$ & $0-100 \mathrm{~A}$ \\
\multicolumn{2}{c}{ Phase adjustment range } & $\leq 0.1 \%$ \\
Phase adjustment fineness & $0 \sim 360.0^{\circ}$ \\
\hline
\end{tabular}

\section{Automatic Test System Design}

5.1 Structure design for the test system

Overall configuration of the test system is shown in Fig.2.

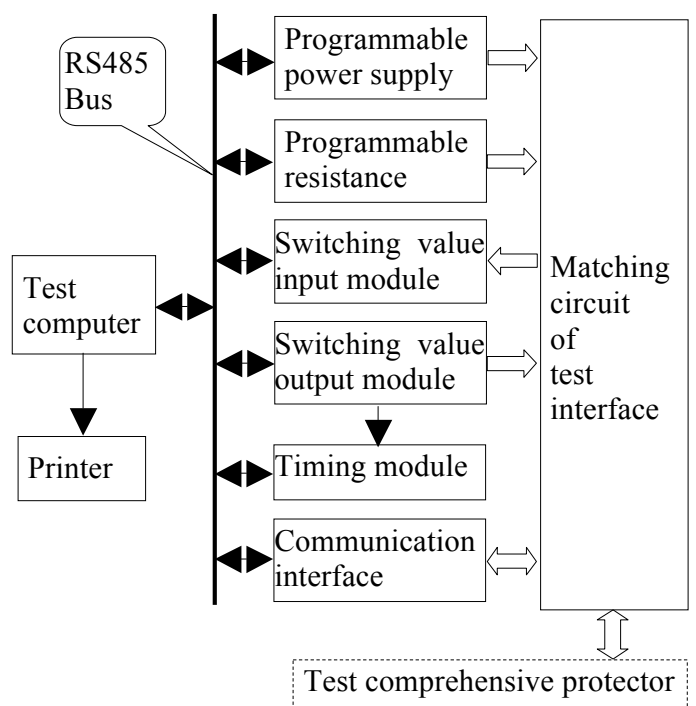

Fig.2 Hardware block diagram of test system 
Programmed input-output testing excitation power supply includes a four-phase voltage source and a threephase current source, used to provide the required power frequency AC voltage and current excitation signal for test. Each phase circuit of voltage and current source is independent, and amplitude, phase, frequency of each phase are independently adjustable.

Programmable resistance simulates the insulation resistance of electrical equipments and loop resistance of the cable monitor, providing the excitation signal for leakage and insulation monitoring protection. Switching value output module provides switching value output signals. In some cases they are used to simulate the external control signals of comprehensive protectors (such as remote opening-closing signals, wind-power block signals, the water level signals, etc.), and in other cases they are used to control timing module for timing. Switching value input module is used to monitor the output response signal of the comprehensive protection. Different types of comprehensive protectors have different types of interface and different number and arrangement of pins. The function of the interface matching circuit is to make sure the same test system interface matches interfaces of different tested comprehensive protectors. The interface matching circuit is one of the necessary measurements to make the test system meet versatility.

In the structure of the test system, on the one hand the test computer is taken as test process controller, which coordinates the various parts of the control system. On the other hand, it also undertakes voltage and current signal occurring and data processing of DDS programmed inputoutput power supply. RS-485 bus hangs the above individual modules on the same communication channel. Then the main test computer is connected to the 485 bus via RS232/RS-485 converter by using a COM port.

\subsection{Test Procedure}

The main procedure for system test is shown in Fig.3.

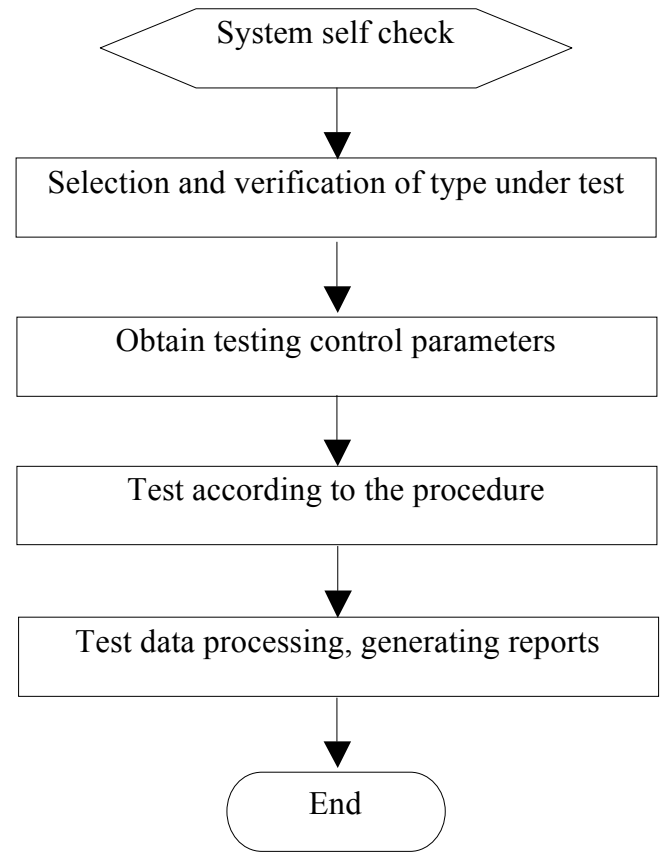

Fig.3 Main flow of test
The specific operation of each item test varies and the general procedure is shown in Fig.4.

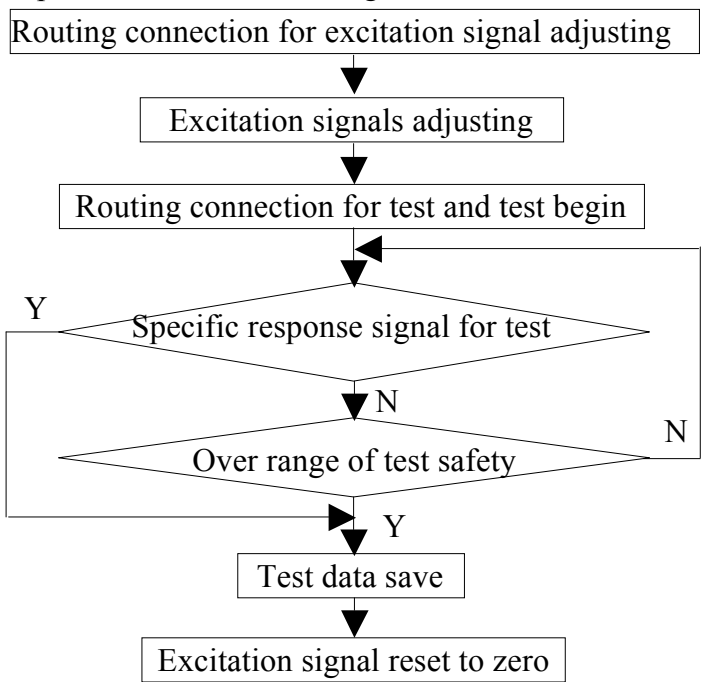

Fig.4 Flow of function test

Table 3 shows the test data of certain ZLZB-5A comprehensive protector tested in the test system. By comparing the test data with the relevant product information it can be concluded that the electrical protection function of the comprehensive protector is qualified.

Table 3 Test report of ZLZB-5A comprehensive protector

\begin{tabular}{|c|c|c|c|}
\hline Type & ZLZB-5A & Number & 2013050401 \\
\hline \multirow{4}{*}{$\begin{array}{l}\text { Leakage } \\
\text { test }\end{array}$} & \multicolumn{2}{|c|}{ Test condition } & test results \\
\hline & \multicolumn{2}{|c|}{ Zero sequence current setting } & $6 \mathrm{~A}$ \\
\hline & \multicolumn{2}{|c|}{ Zero sequence voltage setting } & $10 \mathrm{~V}$ \\
\hline & \multicolumn{2}{|c|}{ Leakage delay setting } & $2 \mathrm{~s}$ \\
\hline \multirow{5}{*}{$\begin{array}{c}\text { Action } \\
\text { resistance }\end{array}$} & \multirow{2}{*}{\multicolumn{2}{|c|}{$\begin{array}{l}\text { Leakage delay value } \\
\text { Insulation monitoring }\end{array}$}} & $1.93 \mathrm{~s}$ \\
\hline & & & $8.44 \mathrm{~K} \Omega$ \\
\hline & \multicolumn{2}{|c|}{ Circuit monitoring } & $0.89 \mathrm{~K} \Omega$ \\
\hline & \multicolumn{2}{|c|}{ Overload tuning multiples } & 0.5 \\
\hline & \multicolumn{2}{|c|}{ Overload test multiples } & 2.0 \\
\hline \multirow{3}{*}{$\begin{array}{c}\text { Overload } \\
\text { test }\end{array}$} & \multicolumn{2}{|c|}{ Delay gear } & 4 \\
\hline & \multicolumn{2}{|c|}{ Test current } & $100 \mathrm{~A}$ \\
\hline & \multicolumn{2}{|c|}{ Action time } & $50.89 \mathrm{~s}$ \\
\hline \multirow{2}{*}{$\begin{array}{c}\text { Short- } \\
\text { circuit test }\end{array}$} & \multicolumn{2}{|c|}{ Short-circuit tuning Multiples } & 3.0 \\
\hline & \multicolumn{2}{|c|}{ Action time } & $0.02 \mathrm{~s}$ \\
\hline \multirow{2}{*}{$\begin{array}{l}\text { Voltage } \\
\text { test }\end{array}$} & \multicolumn{2}{|c|}{ Action of over voltage } & $7142 \mathrm{~V}$ \\
\hline & \multicolumn{2}{|c|}{ Action of under voltage } & $3764 \mathrm{~V}$ \\
\hline
\end{tabular}

\section{Conclusions}

It developed a power frequency programmed input-output testing excitation power supply based on DDS technology, and on that basis it also realized the mining comprehensive protector test system in use. In addition to operations of the connection of comprehensive protectors and test system and some parameter settings must be done manually; other operations can all achieve full automation. By adopting the programmable adjustment of voltage and current excitation signal, it not only improves test efficiency and reduces labor intensity for test, but also meets the technical requirements for test excitation signal and ensures the accuracy of test results.

The design has applied for patent of the People's Republic of China. 
Tian-ye Liu and Zhi-jie Zhang/Journal of Engineering Science and Technology Review 6 (3) (2013) 66 -70

The programmed input-output power supply in this system can also be used for other tests in coal mine electrical laboratory, such as in certificate test of electric energy meters, improving the equipment occupation coefficient.

\section{References}

1. Liu Tian-ye, Zhang Zhi-jie, "Detection technology of coal mine comprehensive protection in use", Industry and Mine Automation 39 (5), 2013, pp. 30-33.

2. Cao Shi-you, "China coal industry encyclopedia (Mechanical and Electrical Volume)", China Coal Industry Publishing House, Beijing, 1997.

3. State Administration of Work Safety, "Rules for coal mine safety", China Coal Industry Publishing House, Beijing, 2010.

4. Fan Rong, "The design of low-voltage vacuum feed switch intelligent comprehensive protector", Mining Safety \& Environmental Protection 34 (4), 2010 pp. 20-24.

5. Wu Zu-wan, Zhang Ya-bin, Song Xiao, "Discussion on necessity of detection for electronic protection plug-ins in mineral electrical equipment", Jiangxi Coal Science \& Technology 29 (1), 2007, pp. 21-22.

6. Chen Tong-bao, Zhang Bo-chao, Huang Jian-qiang, ZHANG Jia, "Detection technology of coal mine electromechanical products", Journal of China Coal Society 35 (11), 2010, pp. 1935-1938.

7. Administration regulations of coal mine safety, "Decree of the State Council No.296", China Coal Industry Publishing House, Beijing, 2000.
8. Borza, P.N, Cotfas, D.T, Cotfas, P.A, Pologea, A, "Improvements on photovoltaic cells test bench system", Journal of Engineering Science and Technology Review, 5 (4), 2012, pp. 38-41.

9. Zhang Wei-guo, Bao Chuan-yu, "Development of MDZD multifunctional test device", Coal Science and Technology 27 (5), 1999, pp. 45-47.

10. Ren Zuo-xin, Ren Jin-biao, "The design of the measuring system for high and low voltage protector", Journal of North China Institute of Technology 24 (1), 2003, pp. 1-4.

11. Li Xiao, "Research of universal test system for mine integrated protecting inserter", North University of China, 2003, 16-18.

12. Li Xiao,Li Lei, "Design of the auto testing system for mine integrated protecting inserter", Chinese Journal of Scientific Instrument 38 (4), 2007, pp. 66-69.

13. Li Yu-shen, "The mine intelligent electronic protection plug-ins testing system and the model", Qingdao University of Science \& Technology, 2008, 1-3.

14. Xia Feng-xian, Chen Xiao-ping, "Development of power frequency inverting AC constant current source", Chinese Journal of Scientific Instrument 29 (8), 2008, pp. 1937-1944.

15. DL/T624-2010, "Technical specifications of test equipment based on micro-computer for protection equipment". 